\title{
Aspectos destacados de las guías europeas de fibrilación auricular
}

\author{
Dr. Juan Manuel Castro ${ }^{1}$
}

\section{Introducción}

La fibrilación auricular (FA) es a nivel mundial la arritmia sostenida más frecuente en la población adulta. A pesar de los crecientes conocimientos acerca de sus mecanismos fisiopatológicos subyacentes, herramientas diagnósticas y terapéuticas, se mantiene como una causa importante de insuficiencia cardíaca (IC), accidente cerebrovascular (ACV) y morbilidad cardiovascular a nivel mundial. Esto se traduce en disminución de la calidad de vida y aumento en la mortalidad de los pacientes, generando elevados costos socioeconómicos.

Las Guías de Práctica Clínica (GPC) para el diagnóstico y manejo de la FA, desarrolladas por la Sociedad Europea de Cardiología en colaboración con la Asociación Europea de Cirugía Cardiotorácica publicadas en el año 2020, sintetizan la evidencia disponible para el manejo de la FA con el fin de facilitar las decisiones clínicas en la práctica habitual ${ }^{(1)}$. Se resumen en el presente texto sus aspectos más destacados.

\section{Diagnóstico de fibrilación auricular}

Las GPC previas recomiendan el diagnóstico de FA en base al análisis de un electrocardiograma (ECG) clásico de 12 derivaciones. Los criterios diagnósticos son: a) un ritmo "irregularmente irregular", b) ausencia de ondas p identificables y c) ausencia de un patrón específico de activación auricular. Las nuevas GPC aceptan el diagnóstico de FA analizando una única derivación electrocardiográfica durante 30 segundos, lo cual refleja la importancia demostrada de las herramientas portátiles de screening, como ser teléfonos inteligentes, relojes inteligentes y aplicaciones móviles.

\section{Clasificación de la fibrilación auricular}

Se mantiene la clasificación previa, que toma como criterio el patrón temporal de inicio y manteni- miento de la arritmia (tabla 1). No obstante, se destaca la recomendación de abandonar el uso de ciertos términos de la clasificación por ser conceptualmente erróneos o aportar confusión a la caracterización de la FA. Más precisamente:

- Fibrilación auricular aislada (o solitaria): actualmente se considera que en todo paciente está presente al menos una causa. Por lo tanto, se recomienda su desuso.

- Fibrilación auricular valvular: el término "valvular" es confuso, ya que hace referencia a la FA en presencia de estenosis mitral moderada-severa o prótesis valvulares mecánicas.

- Fibrilación auricular crónica: se desestimula su uso, al tener definiciones variables en la literatura.

Si bien la carga arrítmica (burden) de FA es de importancia en la caracterización de la enfermedad, el patrón temporal no debería (con excepción del control del ritmo, en especial en agudo) guiar el manejo terapéutico.

Caracterización estructural de la fibrilación auricular: esquema de las $4 \mathrm{~s}$

Teniendo en cuenta los avances en el conocimiento de la FA, se propone el paradigma de la "caracterización estructurada" de la FA, que complementa sin sustituir la clasificación temporal. El esquema analiza cuatro aspectos en forma individual:

- Riesgo de ACV (stroke risk): se recomienda la evaluación del riesgo de ACV trombótico utilizando el score $\mathrm{CHA}_{2} \mathrm{DS}_{2}$-VASc. Un score $\geq 1$ en hombres y $\geq 2$ en mujeres obliga a considerar la anticoagulación a largo plazo en esta población.

- Severidad de los síntomas: la estrategia y los objetivos terapéuticos pueden modificarse según la presencia y severidad de los síntomas relacionados con la FA, que impactan en la calidad de

1. Cardiocentro, Asociación Española. Montevideo, Uruguay.

$\mathrm{El}$ autor declara no tener conflictos de intereses.

Correo electrónico: jmcastrobrugnoli@gmail.com

Recibido Feb 2, 2021; aceptado Abr 12, 2021 
Tabla 1. Clasificación de la FA.

De primer diagnóstico

Paroxística siete días de su inicio.

Persistente

Persistente de larga duración

Permanente

FA sostenida durante al menos siete días, incluyendo episodios que cesan por intervención (cardioversión eléctrica o farmacológica) luego de pasados siete días de su inicio.

FA continua, de más de 12 meses de duración, cuando se decide una estrategia de control del ritmo.

FA aceptada por el paciente y el médico, sin realizar otros intentos de restaurar/mantener el ritmo sinusal. La terapia no debería incluir DAA, ni ablación.

FA: fibrilación auricular; DAA: drogas antiarrítmicas.

vida de los pacientes. Se recomienda el uso de cuestionarios de calidad de vida, así como la escala sintomática de la European Heart Rhythm Association. La utilización de esta escala representa una indicación clase IA de las nuevas GPC.

- Severidad de la carga de FA: se define a la carga de FA como el tiempo total de FA o episodios de frecuencia auricular rápida (atrial high rate episodes [AHRE] detectados por dispositivos intracardíacos) durante un período específico de tiempo. Si bien los principales trabajos suelen evaluar la carga arrítmica durante 24 horas, no está dilucidado el tiempo óptimo de evaluación ni el valor pronóstico de ésta. Aunque la evidencia no es concluyente, los ensayos clínicos aleatorizados (ECA) mostraron de forma consistente un menor riesgo tromboembólico en pacientes recibiendo anticoagulación terapéutica con FA paroxística (menor carga arrítmica) versus pacientes con FA persistente.

- Severidad del sustrato: el sustrato de la FA hace referencia a la presencia de comorbilidades y factores de riesgo cardiovascular y, principalmente, a la dilatación y fibrosis auricular izquierda, causantes de disfunciónn contráctil y anomalías en el acoplamiento electromecánico auricular. Se recomienda la utilizaciónn de técnicas de imagen cardíacas para la caracterización del sustrato mediante ecocardiograma transtorácico (ETT). La ecocardiografía transesofágica (ETE) debería plantearse en pacientes con enfermedad valvular significativa o trombos auriculares conocidos.

Se mencionan como predictores de ACV las siguientes características de la aurícula izquierda: la dilatación, la presencia de contraste espontáneo en el ETE (indicador de estasis auricular), strain alterado, velocidad pico en la orejuela izquierda menor a $20 \mathrm{~cm} /$ segundo y una anatomía diferente a la chicken wing.

La caracterización estructurada con el uso de las $4 \mathrm{~s}$ forma parte de las principales y más aplicables novedades de las nuevas GPC, siendo una recomendación clase IIa.

\section{Cribado poblacional de fibrilación auricular}

Si bien existe escasa evidencia acerca de los beneficios reales de los programas de cribado de FA a nivel poblacional, la FA asintomática se asocia de forma independiente con un mayor riesgo de ACV en comparación con pacientes sintomáticos. Este hallazgo ilustra la importancia de la detección precoz y el tratamiento oportuno de la arritmia. En la última década se han evaluado múltiples dispositivos móviles para el cribado de FA en pacientes asintomáticos. Considerando al ECG de 12 derivaciones como estándar de oro, el uso de aplicaciones en smartphones y relojes inteligentes han demostrado alta sensibilidad y especificidad para su diagnóstico.

Se recomienda el cribado oportunista (mediante la palpación del pulso arterial o la realización de un ECG de 12 derivaciones) en individuos con edad $\geq 65$ años (recomendación clase IB) y el cribado de forma sistemática en pacientes con edad $\geq 75$ años o en presencia de factores de riesgo de ACV.

Debido a la frecuencia de AHRE (10\%-15\% en individuos portadores de dispositivo electrónico) y su relación con la FA subclínica, se recomienda el interrogatorio de los dispositivos intracardíacos para su detección (recomendación IA). 
Tabla 2. Resumen de las principales recomendaciones de la guía.

Principales recomendaciones de la guía Clase Nivel

Se recomiendan los ACOD en preferencia a AVK (excepto válvula protésica mecánica y estenosis mitral moderada-severa).

En pacientes bajo tratamiento con AVK, se recomienda un INR de 2-3, con TRR $\geq 70 \%$. Cuando no se logre este objetivo, cambiar a ACOD asegurando buena adherencia al tratamiento.

El riesgo estimado de sangrado en ausencia de contraindicaciones absolutas para el tratamiento anticoagulante no debe, por sí mismo, guiar las decisiones terapéuticas para el uso de ACO en prevención de ACV.

El patrón clínico de la FA (ver clasificación en tabla 1) no debería condicionar la indicación de ACO.

Se recomiendan los betabloqueantes, diltiazem o verapamilo, para el control de la $\mathrm{FC}$ en pacientes con FA y FEVI $\geq 40 \%$.

Los betabloqueantes y la digoxina se recomiendan para control de la $\mathrm{FC}$ en pacientes con FA y FEVI $<40 \%$.

I

I

III

A

A

B

Para la cardioversión farmacológica de la FA de reciente inicio, se recomienda el uso de vernakalant (en ausencia de SCA o IC severa), o flecainida/propafenona (en ausencia de cardiopatía estructural severa).

Se recomienda la amiodarona intravenosa para la cardioversión farmacológica en pacientes con IC o cardiopatía estructural.

La cardioversión farmacológica solo debe realizarse en pacientes hemodinámicamente estables, luego de considerar el riesgo tromboembólico del paciente.

La cardioversión farmacológica no debe realizarse en pacientes con enfermedad del nodo sinusal, alteraciones de la conducción auriculoventricular o intervalo QT corregido $>500 \mathrm{~ms}$, a menos que el riesgo de proarritmia se haya considerado previamente.

En pacientes con alto riesgo de sangrado (HASBLEED $\geq 3$ ) e indicación de terapia antiplaquetaria, debe considerarse el tratamiento con rivaroxabán 15 $\mathrm{mg} /$ día y dabigatrán $110 \mathrm{mg} / 12$ horas.

En pacientes con SCA e ICP sin complicaciones, se recomienda triple terapia por una semana, continuando posteriormente con clopidogrel y ACO por hasta 12 meses (valorando balance de riesgo entre trombosis del stent y sangrados).

Durante el embarazo, se recomienda la cardioversión eléctrica inmediata en pacientes con inestabilidad hemodinámica o FA preexcitada.

Se recomienda la anticoagulación con heparina o AVK dependiendo del trimestre de embarazo.

Durante el embarazo, se recomiendan los betabloqueantes cardioselectivos (no atenolol) para el control de la FC.

Se recomienda la terapia con amiodarona o betabloqueantes en el perioperatorio de cirugía cardíaca, para prevenir la aparición de FA posoperatoria.

Se debe considerar la terapia con ACO a largo plazo en pacientes con riesgo de ACV que presentan FA en el posoperatorio de cirugía no cardíaca.

Se debe considerar la terapia con ACO a largo plazo en pacientes con riesgo de ACV que presentan FA en el posoperatorio de cirugía cardíaca.

III A

I B

I B

I A

I $\quad \mathrm{A}$

I A

III A

IIa B

B

I A

I C

C

I C

I C

I A

IIa B

$\mathrm{IIb} \quad \mathrm{B}$

ACOD: anticoagulantes orales directos; AVK: antagonistas de la vitamina K; INR: International Normalized Rate; TRR: tiempo en rango terapéutico; ACV: accidente cerebrovascular; FA: fibrilación auricular; ACO: anticoagulación oral; FC: frecuencia cardíaca; FEVI: fracción de eyección del ventrículo izquierdo; SCA: síndrome coronario agudo; IC: insuficiencia cardíaca; ICP: intervención coronaria percutánea. 
Manejo integrado de los pacientes con fibrilación auricular: esquema $A B C$

La estrategia del A (anticoagulación y prevención de ACV, Anticoagulation/Avoid stroke), B (control de síntomas, Better symptom management) y C (optimización cardiovascular y de comorbilidades, Cardiovascular and Comorbidity optimization), se ha asociado a menor riesgo de muerte por todas las causas, muerte cardiovascular, sangrados mayores, ACV y hospitalizaciones en individuos diagnosticados con FA.

\section{A: Anticoagulación y prevención de ACV}

Evaluación del riesgo de ACV. La FA se asocia de forma independiente con un riesgo cinco veces mayor de sufrir un ACV. No obstante, depende de la presencia de varios factores de riesgo identificados en el score $\mathrm{CHA}_{2} \mathrm{DS}_{2} \mathrm{VASc}$, recomendado para guiar la decisión de anticoagulación a largo plazo en pacientes con FA. Si bien algunos scores han demostrado un beneficio en su aplicación (GARFIELD-AF, ABC), su complejidad clínica o la inclusión de biomarcadores dificulta su uso clínico rutinario.

Evaluación del riesgo de sangrado. La evidencia actual indica que el score HASBLED es el mejor predictor de riesgo de sangrado, considerando a un paciente con puntuación $\geq 3$ como de alto riesgo. Sin embargo, no es una contraindicación para el uso de anticoagulantes. Se debe hacer énfasis en tratar los factores modificables y realizar un seguimiento estrecho de los pacientes con factores de riesgo no modificables de sangrado. Se recomienda una reevaluación del riesgo cada 4-6 meses.

Las contraindicaciones absolutas para la anticoagulación oral incluyen sangrados activos significativos, trombocitopenia severa, anemia severa sin causa aclarada o en estudio y hemorragias graves recientes (hemorragia intracraneana $[\mathrm{HIC}]$ ).

Terapia de anticoagulación oral. Los antagonistas de la vitamina K (AVK) continúan siendo el único tratamiento seguro para pacientes con FA en presencia de estenosis mitral reumática o prótesis valvulares mecánicas. El objetivo de llegar a un tiempo en rango terapéutico $\geq 70 \%$ puede ser dificultoso en algunos pacientes, pero en estos valores la eficacia de los AVK es similar a los anticoagulantes no antagonistas de la vitamina $\mathrm{K}$, o anticoagulantes directos (ACOD). Los ACOD no requieren monitorización de rango terapéutico y se asocian a menor riesgo de sangrados significativos. Por lo tanto, se recomienda el uso de ACOD como primera línea en pacientes con FA, a excepción de aquellas personas con FA y estenosis mitral moderada-severa, o portadores de prótesis valvulares mecánicas.
La carga de FA, así como su progresión de FA paroxística a no paroxística, no debería modificar la decisión de iniciar una terapia de anticoagulación oral. La anticoagulación en pacientes con AHRE mayores a 24 horas se encuentra en etapa de evaluación en varios ECA. La terapia antiplaquetaria aislada o en combinación con ACOD, sin otra indicación, está contraindicada por las GPC (recomendación clase III).

Considerando las herramientas de evaluación y la terapéutica disponible se propone, de forma práctica y sintética, tres pasos para la prevención de ACV en pacientes con FA. El primer paso consiste en identificar a los pacientes que no se beneficiarían de la terapia de anticoagulación a largo plazo $\left(\mathrm{CHA}_{2} \mathrm{DS}_{2} \mathrm{VASc}=0\right.$ en hombres, $=1$ en mujeres $)$. El segundo paso es iniciar el tratamiento en individuos con $\mathrm{CHA}_{2} \mathrm{DS}_{2}$ VASc $\geq 2$ en mujeres y $\geq 1$ en hombres. El tercer paso es la elección del fármaco a utilizar (AVK o ACOD), siendo de primera elección los ACOD.

\section{B: Control sintomático de pacientes con fibrilación auricular}

Control de frecuencia cardíaca. El control adecuado de la frecuencia cardíaca mejora la sintomatología y la calidad de vida. Si bien las GPC reconocen la falta de evidencia en cuanto al objetivo ideal de frecuencia cardíaca en la mayoría de los pacientes, se recomienda que sea $<110$ ciclos por minuto. Puede variar en pacientes con taquimiocardiopatía inducida por FA o en ausencia de mejoría sintomática inicial.

Fármacos para control de frecuencia cardíaca. Se recomiendan como de primera línea los fármacos betabloqueantes, antagonistas del calcio no dihidropiridínicos, y la digoxina. Deben tenerse en cuenta algunas consideraciones al momento de elegir el fármaco adecuado para cada individuo:

- Se recomienda el uso de betabloqueantes en los pacientes con IC con fracción de eyección del ventrículo izquierdo (FEVI) reducida. Los fármacos antagonistas del calcio están contraindicados en este contexto. La digoxina no se recomienda en pacientes con un tono simpático-adrenérgico cardíaco aumentado.

- En el contexto agudo, se recomienda el uso de betabloqueantes y antagonistas del calcio por su rápida acción y efectividad en presencia de un tono adrenérgico aumentado, por encima de la digoxina. La combinación de varios fármacos puede ser necesaria.

Ablación del nodo auriculoventricular (NAV). La ablación del NAV con posterior terapia de estimulación cardíaca con marcapasos se ha demostra- 
do como beneficiosa en pacientes con FA permanente y antecedentes de al menos una hospitalización por IC. Se debe considerar esta opción en pacientes que no respondan o no toleren el tratamiento con fármacos para control de frecuencia que no sean candidatos aptos a ablación.

Estrategia de control del ritmo cardíaco. La indicación de restaurar el ritmo sinusal en un paciente con FA es pertinente cuando los objetivos sean reducir la carga sintomática y mejorar la calidad de vida en aquellos pacientes con un adecuado control de frecuencia cardíaca y persistencia de síntomas. Si bien el control del ritmo no ha demostrado ser superior en términos de pronóstico a las estrategias de control de frecuencia, en selectos pacientes el control del ritmo, temprano en la evolución de la enfermedad, puede ser beneficioso y disminuir el sustrato auricular patológico, así como la progresión de la FA. Con el objetivo del control sintomático, la recomendación del control del ritmo es clase IA.

Cardioversión. En pacientes con inestabilidad hemodinámica, la cardioversión eléctrica de emergencia es el tratamiento indicado. En pacientes que se presentan estables, se considera válida la cardioversión eléctrica y la estrategia farmacológica, siendo esta última menos efectiva. El tratamiento previo con fármacos antiarrítmicos puede aumentar la posibilidad de éxito de la cardioversión, además de prevenir recurrencias de la arritmia.

Se ha reportado la no inferioridad de la estrategia "ver y esperar" -watch and wait-con respecto a la mejor oportunidad de realizar la cardioversión. La denominada cardioversión electiva se propone como una opción válida en pacientes con bajo riesgo trombótico y con un inicio de la arritmia menor a 24 horas. Se deben tener en consideración algunos factores que se asocian a una alta tasa de recurrencia de FA tras la cardioversión: mayor edad, sexo femenino, dilatación auricular significativa, y antecedentes de cardioversión previa, enfermedad pulmonar obstructiva crónica, falla renal, enfermedad cardíaca estructural o IC.

La cardioversión eléctrica debe realizarse bajo sedación intravenosa con midazolam, propofol o etomidato. Se requiere una monitorización eléctrica continua, así como de oximetría de pulso. Se recomienda además la disponibilidad de marcapasos externo y drogas, por ejemplo, atropina e isoproterenol, como tratamiento de una eventual bradicardia poscardioversión. La desfibrilación bifásica es de elección, al haber demostrado mayor eficacia que la monofásica.

La cardioversión farmacológica se reserva únicamente para pacientes hemodinámicamente estables, con una eficacia de $69 \%$ dentro de las 48 horas de inicio del tratamiento, siendo más efectiva cuanto menor es el tiempo de inicio de la arritmia. En cuanto a la elección del fármaco, depende de la presencia, tipo y severidad de la enfermedad cardíaca existente. Las drogas antiarrítmicas (DAA) del grupo IC tienen un efecto rápido (3-5 horas), son seguras y con una efectividad superior al $50 \%$ en la reversión a ritmo sinusal. Sus contraindicaciones incluyen la presencia de disfunción ventricular izquierda, hipertrofia del VI, y enfermedad coronaria significativa. La amiodarona es la droga de elección en estos pacientes. Se menciona a vernakalant como droga más efectiva que la amiodarona y la flecainida, pudiendo utilizarse en pacientes con IC o enfermedad coronaria no grave.

En pacientes seleccionados, la estrategia de control de ritmo con fármacos vía oral puede ser elegida. La efectividad es algo menor que la cardioversión en el contexto hospitalario, pero puede ser una opción atractiva, principalmente en pacientes jóvenes, con episodios sintomáticos bien identificados y sin enfermedad cardíaca estructural significativa.

Manejo del riesgo trombótico en pacientes candidatos a control del ritmo. En ausencia de anticoagulación terapéutica previa, y particularmente si el inicio de la arritmia es mayor a 12 horas, existe un riesgo real y significativo de tromboembolismo luego de una cardioversión efectiva. Se recomienda, por lo tanto, la anticoagulación durante tres semanas previas a la cardioversión si el inicio de la arritmia es mayor a 48 horas, o se desconoce. Como alternativa a la anticoagulación previa por tres semanas, la exclusión de trombos cardíacos por ETE permite realizar la cardioversión con seguridad. De identificarse trombos en el ETE, debe asegurarse una anticoagulación terapéutica durante tres semanas previo a la cardioversión, considerando la realización de un nuevo ETE previo al procedimiento (recomendación clase IIa). Ambas estrategias (la anticoagulación durante tres semanas como primera medida o la estrategia ETE y cardioversión eléctrica en ausencia de trombos auriculares) poseen una recomendación clase IIa en las nuevas GPC.

Luego del procedimiento, e independientemente de haberse restaurado el ritmo sinusal, se recomienda la anticoagulación efectiva durante al menos tres semanas. Los fármacos de primera línea para el tratamiento pre y poscardioversión son los ACOD.

Ablación por catéter de la fibrilación auricular. Se ha demostrado la superioridad de la ablación con catéter en comparación al tratamiento con drogas antiarrítmicas en mantener el ritmo sinusal y controlar los síntomas atribuidos a la FA. La indicación 
debe ser individualizada y tener en cuenta los factores que se han asociado a una mayor tasa de recurrencia de FA luego de la ablación: auriculomegalia izquierda, mayor duración de la arritmia, mayor edad, sustrato auricular anómalo (fibrosis y tejido adiposo epicárdico auricular significativos identificados por resonancia magnética cardíaca), insuficiencia renal, y recurrencias tempranas previas (uno de los más potentes predictores). Sin embargo, ningún score ha demostrado predecir de forma robusta el éxito sin recurrencias luego de la ablación.

Si bien se conoce el beneficio sintomático y en cuanto a la mejoría en la calidad de vida, los ECA más importantes que analizaron la mortalidad, eventos cardíacos mayores y ACV asociado a pacientes tratados mediante ablación con catéter, no lograron demostrar su superioridad. No obstante, existe evidencia del beneficio en cuanto a mortalidad y hospitalizaciones de la ablación en pacientes con IC con FEVI reducida. Se recomienda considerar (IIa) como primera línea a la ablación con catéter en este subgrupo de pacientes, lo cual es una novedad de las GPC del año 2020.

Se mantiene, con respecto a las GPC previas, la indicación clase IB de tratar con ablación con catéter a los pacientes en los cuales se sospecha una miocardiopatía inducida por taquicardia.

En el resto de la población la ablación se recomienda como segunda línea luego de al menos un intento fallido de control del ritmo con DAA o en pacientes que requieran y no toleren las mismas (la clase y el nivel de recomendación varían según el patrón temporal y la presencia de factores de riesgo de recurrencia). La ablación con catéter sin el uso previo de DAA (con excepción de los betabloqueantes) posee una clase de recomendación IIa y IIb en pacientes con FA paroxística y con FA persistente sin factores de riesgo de recurrencia, respectivamente.

Las complicaciones del procedimiento se ubican entre $4 \%$ y $14 \%$, graves en $2 \%-3 \%$. La mayoría de las complicaciones ocurren en las primeras 24 horas, siendo la muerte periprocedimiento poco frecuente (menor a $0,2 \%$ ), asociada por lo general a taponamiento cardíaco.

Por último, la ablación de otros sustratos más allá del aislamiento de las venas pulmonares no posee evidencia suficiente como para realizar una recomendación más allá de clase IIb, al menos en todos los pacientes o de forma sistemática.

Tratamiento quirúrgico de la fibrilación auricular. Si bien es efectivo en mantener el ritmo sinusal, no se ha demostrado aún un beneficio de la ablación quirúrgica en cuanto a mortalidad, hospitalizacio- nes, ACV o mejoría en la calidad de vida en pacientes con FA.

El tratamiento quirúrgico concomitante, es decir, en pacientes que serán sometidos a otra cirugía cardíaca, tiene un demostrado efecto beneficioso en aumentar el tiempo libre de arritmia, existiendo escasa evidencia de un beneficio en la incidencia de eventos cardiovasculares en estos pacientes. Debe tenerse en cuenta de forma individualizada la presencia de factores que aumenten el riesgo de recurrencia (se proponen los mismos que para la ablación por catéter). El tratamiento quirúrgico concomitante tiene en las GPC una indicación clase IIa.

La ablación quirúrgica como tratamiento individual (es decir, no concomitante a una cirugía cardíaca por otra indicación) posee beneficios demostrados en tiempo sin recurrencias con respecto a la ablación por catéter. Sin embargo, genera mayores tiempos de hospitalización (y mayores costos económicos), así como una mayor tasa de complicaciones. Se recomienda su consideración en pacientes con FA paroxística o persistente, luego de un intento fallido de control del ritmo con DAA, y considerados como alto riesgo de recurrencia de FA luego de una ablación con catéter (clase IIa).

Con respecto a la anticoagulación en pacientes candidatos a ablación por catéter, se recomienda durante las tres semanas (preferentemente con ACOD) anteriores al procedimiento, sin interrupción de dosis previa. La estrategia de realizar un ETE para descartar trombos auriculares es una alternativa, prefiriéndose aquí la anticoagulación efectiva previa. Luego de la ablación por catéter, se recomienda la anticoagulación durante un mínimo de dos meses a todos los pacientes.

Tratamiento con drogas antiarrítmicas para el control del ritmo a largo plazo. Si bien tiene menor eficacia en mantener el ritmo sinusal que la ablación con catéter, el tratamiento con DAA aumenta el tiempo libre de arritmia con respecto a la ausencia de terapia antiarrítmica, y tienen como beneficio principal la mejoría sintomática.

La elección de la droga antiarrítmica no debe basarse únicamente en la efectividad demostrada, sino también en los posibles efectos adversos, el riesgo proarrítmico, y la presencia y tipo de enfermedad cardíaca en el individuo. Las DAA se han mostrado útiles en disminuir las recurrencias luego de la ablación con catéter, así como luego del tratamiento del ritmo con cardioversión farmacológica y eléctrica.

La amiodarona se recomienda para el control del ritmo a largo plazo en todos los pacientes (incluso aquellos con disfunción del VI), debiéndose considerar otras DAA como primera línea de ser posible (clase IA). 
La flecainida y la propafenona se recomiendan en pacientes sin cardiopatía estructural ni isquémica (clase IA). Si la droga de elección es la flecainida, se debe considerar el tratamiento concomitante con drogas bloqueadoras del NAV (clase IIa).

Se puede considerar el tratamiento con sotalol en pacientes con FEVI conservada y sin cardiopatía isquémica. Se recomienda el monitoreo frecuente del intervalo QT (clase IIb).

\section{C: Control de factores de riesgo cardiovascular y comorbilidades}

La letra $\mathrm{C}$ del esquema propuesto por las GPC incluye la identificación y el manejo de las enfermedades concomitantes, los factores de riesgo cardiovascular y la promoción de un estilo de vida saludable.

Con respecto a las intervenciones del estilo de vida, se recomienda un manejo intensivo del control de peso, lo cual reduce las recurrencias de FA. La obesidad se relaciona, además, con un aumento en las recurrencias de FA luego de la ablación con catéter.

Limitar el uso de alcohol, factor de riesgo de FA incidente y de sangrados significativos en pacientes con FA. Las causas de esto último son múltiples: faltas en la adherencia en el tratamiento anticoagulante, mayor disfunción hepática asociada a la ingesta alcohólica, presencia de várices esofágicas y mayor riesgo de traumatismos.

Se recomienda realizar actividad física de intensidad leve a moderada, evitando el ejercicio extenuante de resistencia (factor de riesgo para la aparición de FA), principalmente en individuos mayores de 50 años.

Debe llevarse a cabo el cribado oportunista de FA en pacientes con apnea obstructiva del sueño, dada la alta prevalencia de la arritmia en esta población. El manejo adecuado de la apnea obstructiva del sueño se relaciona con una mejoría en la incidencia y progresión de la FA, así como en una mejoría sintomática en estos pacientes.

\section{Esquema $A B C$ en poblaciones especiales} Fibrilación auricular en presencia de inestabilidad hemodinámica. Se recomienda la cardioversión eléctrica de emergencia (FA sincopal, edema agudo de pulmón, hipotensión sintomática o shock cardiogénico), y en caso de empeoramiento agudo de los síntomas.

Síndromes coronarios e intervención coronaria percutánea en pacientes con fibrilación auricular. Manejo antitrombótico. Los principales ECA reportan una reducción de sangrados clínicamente significativos y un efecto neutral en eventos cardíacos mayores en pacientes con doble terapia antitrombótica (inhibidor del receptor P2Y12 más ACOD) en comparación con la triple terapia (inhibidor del receptor P2Y12, AVK y ácido acetilsalicílico), luego de una intervención coronaria percutánea (ICP) con colocación de stent.

Si bien existe un mayor riesgo de sangrados mayores en pacientes con triple terapia antitrombótica, debemos poner en contraposición el riesgo de eventos coronarios asociados al implante de stent. Esto obliga a tomar decisiones individualizadas, teniendo en cuenta el balance entre el riesgo trombótico y el riesgo de sangrados mayores en cada paciente.

Indistintamente del plan inicial, se recomienda la doble terapia con un anticoagulante y un fármaco antiplaquetario (de preferencia, clopidogrel) por seis meses luego de la ICP en el contexto de un síndrome coronario agudo, y seis meses en síndromes coronarios crónicos. Se deben evitar ticagrelor y prasugrel, al haberse demostrado un mayor y significativo riesgo de sangrados mayores en pacientes con FA.

Se recomienda, además, evitar la triple terapia en pacientes sometidos a una cirugía de revascularización miocárdica coronaria.

Accidente cerebrovascular y hemorragia intracraneana en pacientes con fibrilación auricular. Los $\mathrm{ACV}$ y las HIC son eventos mayores discapacitantes $y$, en ocasiones, fatales en pacientes con FA. El reinicio temprano de la anticoagulación se asocia con un riesgo significativo de transformación hemorrágica del ACV. Sin embargo, el momento adecuado del reinicio de la anticoagulación luego de un ACV no está definido, ya que la evidencia es escasa. Se recomienda la individualización de la decisión.

Fibrilación auricular posoperatoria. Se define como aquella FA de nueva aparición en el período posoperatorio temprano, con una prevalencia de $20 \%-50 \%$ luego de una cirugía cardíaca, 10\%-30\% en cirugías torácicas y 5\%-10\% luego de cirugías vasculares y colorrectales. El pico de incidencia se encuentra entre el segundo y el cuarto día posoperatorio.

En la tabla 2 se destacan las principales recomendaciones de la guía.

Juan Manuel Castro; ORCID: 0000-0001-6083-5593

Este artículo fue aceptado para su publicación por la Dra. María Victoria Ramos.

\section{Bibliografía}

1. Hindricks G, Potpara T, Dagres N, Arbelo E, Bax JJ, Blomstrom-Lundqvist C, et al. 2020 ESC Guidelines for the diagnosis and management of atrial fibrillation developed in collaboration with the European Association for Cardio-Thoracic Surgery (EACTS). Eur Hear J. 2020;42(5):373-498. doi: 10.1093/eurheartj/ehaa612 